Bundesgesundheitsbl $2011 \cdot 54: 1197-1210$ DOI 10.1007/s00103-011-1347-5

Online publiziert: 22. Oktober 2011

(c) Springer-Verlag 2011

\author{
A. Neumeyer-Gromen ${ }^{1}$ • N. Bodemer ${ }^{1}$ - S.M. Müller ${ }^{2}$ - G. Gigerenzer ${ }^{1}$ \\ ${ }^{1}$ Max-Planck-Institut für Bildungsforschung, Harding Center for Risk Literacy, Berlin \\ 2 Institut für Experimentelle Psychologie, Universität Granada
}

\title{
Ermöglichen Medienberichte und Broschüren informierte Entscheidungen zur Gebärmutterhalskrebsprävention?
}

\section{Hintergrund}

Gesundheitsinformationen aus der Wissenschaft für den Laien können sowohl indirekt über die allgemeinen Medien wie Zeitungen, Internet oder Broschüren als auch direkt kommuniziert werden. Ihre Inhalte unterliegen gleichermaßen der Interpretation und Diskussion durch Wissenschaftler, Journalisten und Laien. Dabei fließen neben den eigentlichen Sachinformationen auch die Interessen und Werte dieser Gruppen ein, woraus sich ausgesprochen kontroverse Betrachtungen ergeben können. Frühere Untersuchungen zeigen erhebliche Defizite bei der Ausgewogenheit der wissenschaftlichen/medizinischen Berichterstattung - zu nennen sind in diesem Zusammenhang beispielsweise überzogene und alleinige Darstellungen des Nutzens einer Maßnahme/Intervention, ohne das Schadenspotenzial zu erwähnen, oder die Angabe missverständlicher Maßzahlen $[1,2$, $3,4,5]$. Ausgewogenheit ist jedoch unerlässliche Voraussetzung für eine informierte Entscheidung des Laien für oder gegen die Teilnahme an einer Präventions- oder Behandlungsmaßnahme. Die Möglichkeit zur Partizipation durch Informationen gebieten die ethischen Verpflichtungen eines demokratisch-pluralistischen Gesellschaftssystems. Ob im konkreten Fall aber partizipatorische oder direktive Entscheidungsprozesse bevorzugt werden, obliegt dem Einzelnen.

Nun stellt sich die Frage nach der Definition einer ausgewogenen Berichterstattung. Die Illusion der Gewissheit $[6,7,8]$ ebnet hier die Antwort: Demnach sind Interventionen nicht durch die Dichotomie von Vor- oder Nachteilen, sondern durch beides charakterisiert. So sind alle Maßnahmen mit Unsicherheiten behaftet, die Entscheidungen unbequemer machen. Dies liegt an den probabilistischen Schätzungen in der Epidemiologie. Sie beziehen sich auf Populationen und beschreiben ein Ereignis als mehr oder weniger wahrscheinlich, liefern aber keine Gewissheit. Statistische Aussagen führen in das Dilemma, dass sie sich auf einem Kontinuum befinden und nicht determinieren können, wen das Ereignis im Einzelfall trifft. Demgegenüber sind der Ereigniseintritt für den Betroffenen sowie die Entscheidungen des Individuums und der zuständigen Public-Health-Akteure dichotom (ja versus nein) [8]. Bei allen medizinischen Interventionen sind Unsicherheiten abzuwägen.

Folglich liegt der erste Schritt zu einer ausgewogenen Berichterstattung in der Beantwortung der Frage nach den Vorund Nachteilen einer Intervention/Maßnahme. Beispielsweise kann ein Brustkrebsscreening nicht nur dazu beitragen, die Morbidität und Mortalität aufgrund der rechtzeitigen Behandlung früh erkannter Krebserkrankungen zu senken, sondern durchaus auch Nachteile haben $[9,10]$ : Denn falsch-positive Befunde, die für eine davon betroffene Person mit unnötigen Ängsten und invasiven Fehlbehandlungen einhergehen können, sind ein erheblicher Preis dafür, dass auf der anderen Seite Frauen vor dem Brustkrebstod gerettet werden. Um hier abwägen zu können, müssen das Individuum und $\mathrm{Pu}$ blic-Health-Akteure über entsprechend ausgewogene Informationen verfügen.

Neben den Inhalten haben auch die in einer Berichterstattung gewählten Formate erheblichen Einfluss auf den Informationsgewinn. So ist die Darstellung natürlicher statt relativer Häufigkeiten ein maßgebliches Kriterium für Verständlichkeit [7]. Eine Risikoverminderung um 25\% kann sowohl einen Interventionseffekt von vier auf drei Krankheitsbetroffene pro zehn Personen als auch einen von vier auf drei Betroffene pro 1.00o.ooo Personen bedeuten, wobei Letzteres ungleich unbedeutender ist als der erste Effekt. Die Angabe absoluter Zahlen mit Bezug zur Grundgesamtheit ist daher deutlich transparenter als die relativer Effektmaße und wird von internationalen Wissenschaftsorganisationen wie der Cochrane Collaboration inzwischen auch gefordert [11].

Am Beispiel der Zervixkarzinomprävention untersuchen wir anhand der $\mathrm{Hu}$ manen-Papillom-Virus (HPV)-Impfung und des Papanicolaou (Pap)-Screenings, welche entscheidungsrelevanten Inhalte und wie sie hinsichtlich Vollständigkeit (Nutzen und Schaden), Transparenz (natürliche Häufigkeiten) und Richtigkeit in den Medien und öffentlichen Informationsmaterialien abgebildet werden. Das Beispiel hat hohe Public-Health-Relevanz, da es potenziell alle Mädchen im Alter zwischen zwölf und 17 und alle Frauen ab 20 Jahren betrifft. Die Empfehlung zur HPV-Impfung im Jahr 2007 hat eine große journalistische und öffentliche Resonanz erzeugt, die durch die Verleihung 
des Nobelpreises an den HPV-Forscher Professor zur Hausen im Jahr 2008 nochmals verstärkt wurde. Von den zwei verfügbaren HPV-Impfstoffen haben wir beispielhaft Gardasil $^{\circledR}$ ausgewählt, da er früher zugelassen wurde und einen größeren Marktanteil hat.

In der vorliegenden Studie werden sowohl Medienberichte und Informationsmaterialien zu einer Innovation (HPVImpfung) als auch zu einem etablierten Verfahren (Pap-Screening) untersucht. Ziel ist es zu prüfen, ob diese Materialien informierte Entscheidungen für oder gegen die individuelle Teilnahme an diesen Präventionsmaßnahmen und für oder gegen ihren flächendeckenden Einsatz auf Public-Health-Ebene ermöglichen.

\section{Methode}

Vor der Medienanalyse wurden als Referenz sogenannte „Facts-Boxes“ mit wesentlichen wissenschaftlichen Fakten zur HPV-Impfung und zum Pap-Screening erarbeitet. Es handelt sich um vereinfachte, kompakte Präsentationsformate, die das Verständnis von Gesundheitsinformationen verbessern [12].

Die Impfung als Primärprävention zur Verhinderung von Erkrankungen ist vom Screening als Sekundärprävention zur Früherkennung bereits vorhandener Erkrankungen zu unterscheiden. Der potenzielle Nutzen und Schaden wird bei der Impfung direkt anhand von Morbiditäts- oder Mortalitätsveränderungen abgebildet. Beim Pap-Test erfolgen zwei Bewertungsschritte: Erfasst werden die Testgenauigkeit und die sich daraus ergebenden Folgen sowie der Effekt der Früherkennung auf die Krankheitslast, was die Beurteilung von Screening-Programmen komplexer macht.

\section{Facts-Box zur HPV-Impfung}

Um den Wirkmechanismus und die Effektivität einer HPV-Impfung und in diesem Zusammenhang gemachte Aussagen zu Wahrscheinlichkeiten zu verstehen ( $\bullet$ Abb. 1), bedarf es grundlegender Kenntnisse über die Ätiologie von Zervixkarzinomen, die HPV-Virusdynamik, die Pathologie und spontanen Rückbildungsraten von HPV-In- fektionen und Krebsvorstufen (Dysplasien) $[13,14,15,16]$, der Ansätze und Empfehlungen zur Prävention von Zervixkarzinomen sowie der sogenannten Basisrate $[17,18,19,20]$ : Die Basisrate gibt die grundlegende Wahrscheinlichkeit an, überhaupt an einem Zervixkarzinom zu erkranken. Um diesbezügliche Zahlenangaben einordnen zu können, sind Vergleichszahlen zu anderen bekannten Krankheitsentitäten (hier alle Krebserkrankungen) erforderlich. Es folgen in der HPV-Facts-Box dann Angaben zum Nutzen [21, 22, 23, 24], zu den Nebenwirkungen [23, 24, 25] und den Kosten [26, 27, 28] der HPV-Impfung. Diese basieren auf den zum Zeitpunkt der Impfeinführung verfügbaren Daten aus dem Zulassungsverfahren für Gardasil ${ }^{\oplus}$ und auf dem orientierenden extrapolierten Präventionspotenzial. Letzteres beruht auf optimistischsten Annahmen (100\%ige Wirksamkeit der Impfung gegen persistierende HPV-16/18-Infektionen, die die Ursache für $70 \%$ aller Zervixkarzinome sind; Teilnahmerate von $85 \%$; lebenslange Immunität [29]).

Die erste Zahl in der HPV-Box zum Nutzen der HPV-Impfung, das heißt die dort genannte Effektivität von 27\% (Auftreten gefährlicher Vorstufen von Gebärmutterhalskrebs bei 20 von 1000 geimpften Frauen im Vergleich zu 28 von 1000 nicht geimpften Frauen [21]), simuliert die Jungfräulichkeit durch Negativtestung auf HPV 16 und 18 zu Studienbeginn, die der aktuellen Impfempfehlung entspricht, lässt aber spätere Protokollverletzungen, wie zum Beispiel das Auslassen einer Impfung, zu, was der Impfrealität entspricht. Die zweite Effektivitätszahl von 13,5\% (Auftreten gefährlicher Vorstufen von Gebärmutterhalskrebs bei 42 von 1000 geimpften Frauen im Vergleich zu 49 von 1000 nicht geimpften Frauen [21, 22, 23]) reflektiert, dass eine HPV-Infektion zu Studienbeginn möglich sein kann, da auch in der Impfpraxis nicht auf das Vorliegen einer Infektion getestet wird. Dabei sind durch Umrechnung auf 1000 Teilnehmer Rundungsunterschiede zu den ursprünglichen Studienzahlen zu berücksichtigen.

Die Zahlen beziehen sich auf gefährliche Krebsvorstufen (Dysplasien, Neopla- sien) der Schwere von mindestens CIN2+ (gemäß WHO-Definition, CIN: zervikale intraepitheliale Neoplasien) als Surrogatparameter (und nicht auf die Endstufe "Zervixkarzinom“). Sie beziehen sich auf alle Krebsvorstufen, die von kanzerogenen HPV-Typen (also nicht nur von HPV 16/18) verursacht sein können, da die Reduktion der Gesamtdysplasierate maßgeblich ist, um eine Vorstellung über den Effekt der Impfung auf die Krankheitslast zu erhalten. Zwar ist bei Einhaltung des Studienprotokolls eine sehr hohe Schutzwirkung der Impfung (bis nahezu $100 \%$ ) vor HPV-16/18-spezifischen Dysplasien in der Idealpopulation zu verzeichnen, für eine Näherung an Populationseffekte in der realen Versorgungssituation ist gemäß der Europäischen Arzneimittelagentur (EMA) aber die Betrachtung aller, unabhängig vom HPV-Typ verursachter Dysplasien sowie die Berücksichtigung möglicher Studienprotokollabweichungen entscheidend. Die Angaben beziehen sich auf die zu Beginn des Suchzeitraums verfügbaren Daten aus der Zulassung und auf die daraus resultierende Impfempfehlung; sie sind nach dem derzeitigen Veröffentlichungsstand weiterhin aktuell [30, 31].

\section{Facts-Box zum Pap-Screening}

Die Angaben zum Nutzen des Pap-Screenings (- Abb. 2) entstammen ökologischen Daten aus allgemeinen Bevölkerungsstatistiken, die seit Einführung des Screenings 1971 gesammelt wurden [17, 32, 33]. Ergebnisse aus randomisiert-kontrollierten Studien liegen nicht vor. Den Angaben zur Ätiologie und zur Basisrate des Zervixkarzinoms liegen die gleichen Quellen wie in der Box zur HPV-Impfung zugrunde.

Bei Screeninguntersuchungen ist nicht nur die Reduktion der Morbiditäts- und Mortalitätsraten, sondern auch ihre Testgenauigkeit von besonderer Bedeutung, da hier per definitionem symptomlose Personen untersucht werden, von denen die meisten auch tatsächlich gesund sind, aber aufgrund möglicher falsch-positiver Befunde Gefahr laufen, fehlbehandelt zu werden [ $[17$, 32, 33]. Im internationalen Durchschnitt ist für die Neoplasiestadien CIN1 bis $3+$ 
eine Screening-Sensitivität von 50\% und für die Stadien CIN2/3 eine von 50 bis $70 \%$ bei einer Spezifität von $95 \%$ anzunehmen [33, 34]. Diesen Daten werden die Zahl der durchgeführten Pap-Untersuchungen, der verdächtigen Befunde, die Zahl resultierender Operationen $[17,32,33]$ und die Kosten für das Screening gegenübergestellt $[35,36]$.

\section{Medienanalyse zur \\ Laien-Kommunikation}

\section{Suchstrategie, Ein- und Ausschlusskriterien}

Es wurden alle deutschen Berichte aus Arztpraxen, aus der LexisNexis-Datenbank und aus dem Internet herangezogen, die allgemein- beziehungsweise laienverständliche Informationen $\mathrm{zu}$ Gardasil $^{\varpi}$ oder zum Pap-Screening enthielten, soweit sie nicht Kurzmeldungen (<200 Worte), Impfkalender ohne weitere Erläuterungen über Impfung und Screening oder wissenschaftliche Literatur darstellten. Im Zeitraum zwischen Februar bis Mai 2009 wurde in allen kinderärztlichen und gynäkologischen Praxen von Berlin-Kreuzberg und Berlin-Wilmersdorf nach Broschüren zur HPV-Impfung gefragt. Nach Informationsmaterialien zum Pap-Screening wurde nur in frauenärztlichen Praxen gefragt, da es nur für Erwachsene empfohlen wird.

Die Zeitungsartikel wurden über LexisNexis, der umfangreichsten Datenbank für deutsche Pressemeldungen, erfasst. Anhand von Online-Enzyklopädien wurden die verschiedenen Synonyme für die HPV-Impfung und das Pap-Screening zusammengetragen. Daraus resultierte ein sensitiver Suchstring mit Begriffskombinationen ( $\bullet$ Tab. 1), der für die Datenbankrecherche zwischen März 2007 und Mitte Juni 2009 eingesetzt wurde. Die Kopplung mit dem zweiten Impfstoff Cervarix ${ }^{\circledast}$ im Suchstring ist dem breiten Suchansatz geschuldet, da auf Cervarix $^{\circledast}$ in verschiedenen Berichten ergänzend hingewiesen wird, sodass Berichte $\mathrm{zu}$ Gardasil ${ }^{\triangleright}$ auch darüber identifiziert werden konnten.

Zur Erfassung relevanter Webseiten wurde von Januar bis Mai 2009 je-

Bundesgesundheitsbl 2011 · 54:1197-1210 DOI 10.1007/s00103-011-1347-5

(c) Springer-Verlag 2011

\section{A. Neumeyer-Gromen · N. Bodemer · S.M. Müller · G. Gigerenzer Ermöglichen Medienberichte und Broschüren informierte Entscheidungen zur Gebärmutterhalskrebsprävention?}

\section{Zusammenfassung}

Die Zervixkarzinomprävention hat mit Einführung und Empfehlung der neuen Humanen-Papillom-Virus (HPV)-Impfung im Jahr 2007 große öffentliche Aufmerksamkeit erlangt. Kann sich die Öffentlichkeit auf Basis der Auskünfte von Medien und Broschüren zur Zervixkarzinomprävention informiert entscheiden, ob sie an dieser teilnehmen will oder nicht? Um diese Frage zu beantworten, wurde von 2007 bis 2009 eine Medienanalyse zur HPV-Impfung (Gardasi ${ }^{\odot}$ ) und zum Papanicolaou (Pap)-Screening in Deutschland durchgeführt. Geprüft wurde hier, ob die Mindestanforderungen an die Vollständigkeit (Darlegung des Nutzens und Schadens der Intervention), Transparenz (Angabe absoluter Zahlen) und Richtigkeit (Bezug zum Outcome, bestehende Unsicherheiten, Zahlengröße) für medizinische Informationen in der Laienkommunikation erfüllt waren. Als Referenz wurden vorab Facts-Boxes mit den für die jeweilige Präventionsmaßnahme erforderlichen Kennzahlen erarbeitet (Epidemiologie der HPV-Infektionen und des Zervixkarzinoms, Testgüte, Wirksamkeit, Nebenwirkungen, Kosten). Obwohl alle Medien und Broschüren zur HPV-Impfung ihre Präventions- wirkung anführten, nannten nur 41\% Zahlen zur Effektivität ( 90 von 220 Materialien) und $2 \%$ machten absolute Zahlenangaben zur Risikoreduktion (fünf von 220 Materialien) für den Krebssurrogatparameter "Dysplasie", von der aber keine richtig war. Einmal wurde das Präventionspotenzial richtig wiedergegeben; 48\% (105 von 220 Materialien) erwähnten Vor- und Nachteile der Impfung. 20\% der Materialien zum Pap-Screening präsentierten Zahlen zur Testgüte (vier von 20 Materialien) mit einer absoluten Zahlenangabe und $25 \%$ Zahlen zum Präventionspotenzial (fünf von 20 Materialien), die alle korrekt waren. $25 \%$ wiesen auf die Möglichkeit falsch-positiver Befunde hin (fünf von 20 Materialien). Die Mindestanforderungen an medizinische Informationen für die Laienkommunikation erfüllten für die HPV-Impfung eines von 220 und für das Pap-Screening eines von $20 \mathrm{Ma}-$ terialien. Informierte Entscheidungen auf Basis der Berichterstattung sind also derzeit kaum möglich.

Schlüsselwörter

Gebärmutterhalskrebs · HPV-Impfung . Pap-Screening $\cdot$ Medienanalyse $\cdot$ Facts-Box

\section{Do media reports and public brochures facilitate informed decision making about cervical cancer prevention?}

Abstract

With the introduction and recommendation of the new HPV (human papillomavirus) vaccination in 2007, cervical cancer prevention has evoked large public interest. Is the public able to make informed decisions on the basis of media reports and brochures? To answer this question, an analysis of media coverage of HPV vaccination (Gardasi ${ }^{\circledR}$ ) and Pap (Papanicolaou) screening was conducted from 2007-2009, which investigated the minimum requirement of completeness (pros and cons), transparency (absolute numbers), and correctness (references concerning outcome, uncertainty, magnitude) of the information. As a bench mark, facts boxes with concise data on epidemiology, etiology, benefits, harms, and costs were compiled in advance. Although all vaccination reports and brochures covered the impact of prevention, only $41 \%$ provided concrete numbers on effectiveness
(90/220) and $2 \%$ on absolute risk reductions for the cancer surrogate dysplasia $(5 / 220)$, whereby none of the latter numbers was correct. The prevention potential was correctly presented once. Only 48\% (105/220) mentioned pros and cons. With regard to screening, 20\% (4/20) provided explicit data on test quality and one expressed these in absolute numbers, while $25 \%(5 / 20)$ reported the prevention potential; all given numbers were correct. Finally, 25\% (5/20) mentioned the possibility of false positive results. Minimum requirements were fulfilled by $1 / 220$ vaccination and $1 / 20$ screening reports. At present, informed decision making based on media coverage is hardly possible.

Keywords Cervical cancer $\cdot \mathrm{HPV}$ vaccination $\cdot$ Pap screening $\cdot$ Media analysis $\cdot$ Facts box 


\section{Originalien und Übersichten}

\section{Die HPV-Impfung mit Gardasil ${ }^{\circledR}$}

Was ist das Ziel der Impfung? ${ }^{[19]}$

Wie werden HPV-Viren übertragen?[19]

Welche möglichen Folgen hat eine Infektion? ${ }^{[13-16]}$

Wie häufig ist Gebärmutterhalskrebs in Deutschland?[20]

Todesfälle an

Gebärmutterhalskrebs

allen Krebsarten

Erkrankungsfälle an Gebärmutterhalskrebs

$$
\text { allen Krebsarten }
$$

Können Infektionen und Krebsvorstufen ohne Behandlung

abheilen?[13,15,16]

Für wen wird die Impfung empfohlen?[19,31]

Wie lange hält der Impfschutz an? ${ }^{[19,31]}$

Können andere HPV-Typen, gegen die nicht geimpft wurde,

nach der Impfung verstärkt und/ oder abgeschwächt

auftreten?[19]

Welche anderen Vorbeugemöglichkeiten sind bekannt? $[18,19]$
Infektionen mit Humanen Papillomviren (HPV) Typ 16 und 18 vorzubeugen, um das Vorkommen von Gebärmutterhalskrebs zu senken. Zusätzlich Schutz gegen Genitalwarzen.

Durch intime Sexualkontakte.

Infektionen durch potenziell 18 verschiedene HPV-Typen können über Jahrzehnte hinweg zu Gewebeveränderungen in Form von 1.) Krebsvorstufen am Muttermund führen, die dann 2.) Gebärmutterhalskrebs auslösen können. 70 von 100 Gebärmutterhalskrebsfällen sind durch HPV 16/18 verursacht.

$$
\text { von 100.000 Frauen pro Jahr }
$$

alle Frauen pro Jahr

$\begin{array}{rr}3 & 1.500 \\ 230 & 101.000 \\ 15 & 6.200 \\ 500 & 200.000\end{array}$

Ja. Zur Orientierung dienen folgende Angaben: Spontane Abheilungen kommen bei Infektionen in über 90 von 100 Fällen und bei Krebsvorstufen in über 50 von 100 Fällen vor.

Generell für Mädchen im Alter von 12 bis 17 Jahren möglichst vor den ersten intimen Sexualkontakten. Für ältere Frauen kann im Einzelfall eine Impfung mit dem/der behandelnden Arzt/Ärztin erwogen werden.

Mindestens 5 Jahre.

Möglicherweise ja. Es handelt sich um theoretische Überlegungen, die Replacement und Kreuzimmunität genannt werden, ohne dass dies bislang abschließend beantwortet werden kann.

Krebsfrüherkennung mit Papanicolaou/Pap-Test ab dem 20. Lebensjahr, die auch nach einer Impfung weiterhin genutzt werden sollte. Der regelmäßige Kondomgebrauch kann 7 von 10 HPV-Infektionen verhindern.
Welchen Nutzen zeigt Gardasil in den wissenschaftlichen Studien?*

$$
\begin{aligned}
& \text { von 1.000 Frauen } \\
& \text { geimpft nicht geimpft }
\end{aligned}
$$

1.) Auftreten gefährlicher Vorstufen von Gebärmutterhalskrebs (durch alle HPV-Viren) Teilnehmerinnen zu Studienbeginn nicht mit Impfstofftypen infiziert/Jungfrauen[21]

Abb. $1 \Delta$ Facts-Box zur HPV-Impfung 
Welchen Nutzen kann die Impfung in Deutschland zusätzlich zum Pap-

Test eventuell entfalten? ${ }^{[29]}$

Todesfälle

Erkrankungen

$$
\text { mit Impfung }
$$

ohne Impfung

$$
\text { mit Impfung }
$$

ohne Impfung von 1.000 Frauen

$$
\text { von 100.000 Frauen }
$$

pro Jahr

auf Lebenszeit

2

3

7

10

pro Jahr
2

3

11

15 alle Frauen

pro Jahr

1.080

1.500

4.520

6.200

Hat die Impfung mit Gardasil ${ }^{\circledR}$ Nebenwirkungen? ${ }^{[23,25]}$ Auf Basis des europäischen Zulassungsverfahrens:

$$
\text { Sehrhäufig - häufig }
$$

$1.000-\geq 10.000$ von 100.000

- Fieber; Injektionsstelle: Rötung, Schmerz,

Schwellung, Bluterguss, Juckreiz

$$
\begin{aligned}
& \text { Gelegentlich - selten } \\
& 10-1.000 \text { von } 100.000
\end{aligned}
$$

- unspezifische Gelenkentzündung, Gelenk-

beschwerden

- Nesselsucht (schwere allergische Reaktion)
Sehr selten

$<10$ von 100.000

- Verengung der Atemwege mit

schwerer Luftnot

Auf Basis von Spontanmeldungen nach der Zulassung aus einer Population, deren Größe und Dunkelziffer unbekannt ist. Die Meldungen stehen in zeitlichem Zusammenhang mit der Impfung; ob sie dadurch verursacht wurden, bleibt unklar:

- schwere neurologische Erkrankung (Guillain-Barré-Syndrom), Lähmungserscheinungen, Gesichtsnervlähmung, Krampfanfall

- Erbrechen, Muskelschmerzen, Lymphknotenschwellung, allergische Überempfindlichkeitsreaktionen

- vereinzelt Todesfälle

\section{Wie hoch sind die Kosten für die Impfung in Deutschland? ${ }^{[26-28]}$}

Kosten für eine Grundimmunisierung

Gesamtkosten für einen Mädchenjahrgang

ca. 200 Mill. Euro

Jährliches Präventionsbudget der Gesetzlichen Krankenkassen für alle Versicherten und alle Erkrankungen

* Die Zahlen der ersten Nutzenangabe in der Tabelle sind auf S. 1922, 2. Abschnitt in der Future-II-Studie zu finden; dies sind die Ergebnisse der Auswertung der a-priori definierten „Unrestricted Susceptible Population“, die mit der kombinierten Auswertung von Future I und Future II als MITT-2-Analyse im EMA-Report 2008 auf S. 22 Tabelle 15 vergleichbar ist [23]. Die Zahlen der zweiten Nutzenangabe sind auf S. 22, letzter Abschnitt und in Tabelle 15 als „MITT-3" -Analyse zu finden und entsprechen der a-priori definierten „Modified Intention-To-TreatAnalyse; es handelt sich um eine kombinierte Auswertung aus Future I und Future II [23].

\section{Abb. $1 \Delta$ Fortsetzung}




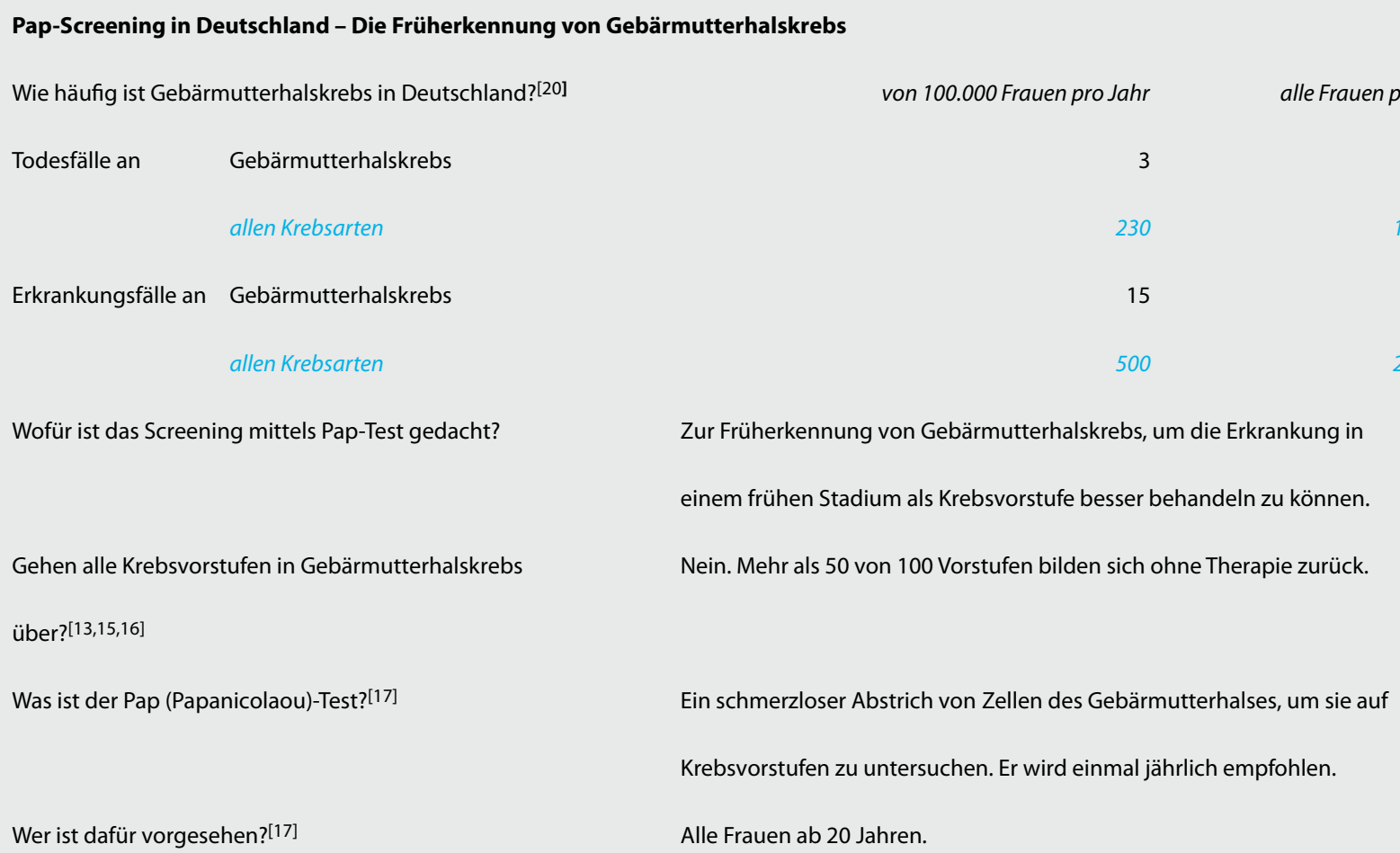

Erkrankungsfälle an Gebärmutterhalskrebs

Wofür ist das Screening mittels Pap-Test gedacht?

Gehen alle Krebsvorstufen in Gebärmutterhalskrebs über?[13,15,16]

Was ist der Pap (Papanicolaou)-Test? ${ }^{[17]}$

Wer ist dafür vorgesehen? ${ }^{[17]}$

Zur Früherkennung von Gebärmutterhalskrebs, um die Erkrankung in einem frühen Stadium als Krebsvorstufe besser behandeln zu können. Nein. Mehr als 50 von 100 Vorstufen bilden sich ohne Therapie zurück.

Ein schmerzloser Abstrich von Zellen des Gebärmutterhalses, um sie auf Krebsvorstufen zu untersuchen. Er wird einmal jährlich empfohlen.

Alle Frauen ab 20 Jahren.

Wie genau unterscheidet der Pap-Test hochgradig krebsgefährdete von gesunden Frauen? [17]

1.000 Frauen

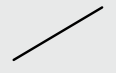

krebsgefährdet

10
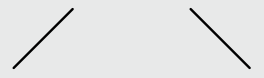

Test richtig positiv

7 Test falsch negativ

3

50

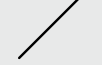

Test falsch positiv

Test richtig negativ

940 


\begin{abstract}
(Herausschneiden eines Kegels am Gebärmutterhals; kann später zu Schwangerschaftskomplikationen führen), seelische Folgen/Angst
Von 1000 Frauen, die mittels Pap-Test untersucht werden, laufen 10 Frauen Gefahr, Krebs zu entwickeln. Von diesen 10 Frauen werden 7 als gefährdet erkannt. Von den Gesunden werden 50 als gefährdet eingeschätzt, obwohl sie es nicht sind (Fehlalarm). Von den 57

Frauen, die positiv getestet wurden, sind nur 7 Frauen tatsächlich gefährdet (12 von 100). So führen nicht nur die 7 richtig erkannten Tests, sondern auch die 50 Fehlalarme gleichermaßen zu weiteren einschneidenden Maßnahmen, obwohl sie gesund sind.
\end{abstract}

In welchem Ausmaß kann das Pap-Screening in Deutschland

Krebs verhindern?

Todesfälle an Gebärmutterhalskrebs

Erkrankungsfälle an Gebärmutterhalskrebs

mit Screening ${ }^{[20]}$

ohne Screening $[32,33]$

\section{von 1.000 Frauen}

auf Lebenszeit

mit Screening ${ }^{[20]}$

ohne Screening ${ }^{[32,33]}$
3

10

10

30

\section{von 100.000 Frauen}

pro Jahr

3

6

15

40 alle Frauen

pro Jahr

1.500

3.000

6.200

16.600

Wie viele Untersuchungen/ Operationen werden dazu

durchgeführt? ${ }^{[17]}$

Pap-Tests

\title{
Abb. $2 \triangle$ Fortsetzung
}

de Homepage der in $\bullet$ Tab. 2 genannten Einrichtungen einschließlich ihrer Linksammlungen durchsucht. Die Suchfunktion der jeweiligen Homepage wurde mit den Begriffen „HPV UND Impfung ODER Humane Papillomvirus Impfung ODER Papanicolaou ODER Pap-Abstrich" genutzt.

\section{Inhaltsanalyse, Kodierungsschema, Auswertung}

Die Medienanalyse folgte einem inhaltsanalytischen Ansatz [37], mit dem festge- stellt werden soll, ob überhaupt, wie häufig und auf welche Weise bestimmte Inhalte in den allgemeinen Medien für eine informierte Entscheidung von Laien kommuniziert werden. Zu diesem Zweck wurden die folgenden vier Kodiermodule definiert:

Das „allgemeine Kodiermodul“ erfasste Angaben zum Veröffentlichungsdatum, zur Beitragsart (Kurzbeitrag, ausführlicher Bericht) und zur Art des Mediums (Broschüren, Zeitungsartikel, Webseiten). Zudem wurden die Verwendung von Illustrationen (zum Beispiel Visuali- sierungen von Zahlen oder Fotos) und der Beitragsstil (werbend, warnend, ausgewogen) kodiert. Hiervon ist das „Akteurmodul" $\mathrm{zu}$ unterscheiden, in dem Verfasser, Akteure (Gesundheitsprofessionelle oder Laien als Wissensvermittler) und Zielgruppen (betroffene Laien, Allgemeinbevölkerung oder Professionelle) vermerkt wurden. Im dritten Modul „Risiko-Ursachen" wurde geprüft, ob Informationen zur Epidemiologie und Ätiologie des Gebärmutterhalskrebses, das heißt zu seiner Häufigkeit und seinen Ursachen vorlagen, und ob der diesbezügliche Präventionsan- 


\section{Tab. 1 Suchstring für die Datenbankrecherche in LexisNexis}

\section{HPV-Impfung}

(hpv! oder papillom! oder kondylom! oder condylom! oder dna-vir! oder hp-vir! oder genitalwarz! oder warzenvir! oder gebärmutterhalsk! oder gebärmutterhalst!) und (impf! oder vakzin! oder immunisierung oder protektiv oder protektion oder prävention oder vorbeugung oder gardasil oder sanofi pasteur oder cervarix oder zervarix oder glaxo smithkline oder silgard oder merck)

\section{Früherkennung von Gebärmutterhalskrebs mittels Pap-Abstrich}

((gebärmutterhals! oder zervix! oder cervix! oder muttermund! oder kollum! oder genital!) und (karzinom oder carcinom oder maligne oder krebs oder tumor oder geschwulst oder geschwür)) und (pap-test oder papanicolaou oder diagnostik oder pap-abstrich oder zellabstrich oder nomenklatur oder Krebsvorstufe oder vorstufe oder präkanzerose oder frühkarzinom oder dysplasie oder früherkennung oder screening oder sekundärprävention oder prävention)

Tab. 2 Bei der Internetsuche berücksichtigte Gesundheitsinstitutionen

Behördliche Einrichtungen/Medizinische Selbstverwaltung im deutschen Gesundheitswesen

Bundesministerium für Gesundheit (BMG)

Bundeszentrale für gesundheitliche Aufklärung

Robert Koch-Institut

Paul-Ehrlich-Institut

Gemeinsamer Bundesausschuss

Krankenkassen und deren Bundesverbände

Bundesärztekammer und alle Landesärzte-

kammern

Landesgesundheitsministerien und Landesgesundheitsämter

Bundesbeauftragte der Bundesregierung für die Belange der Patientinnen und Patienten

Pharmazeutische Impfhersteller

Sanofi Pasteur

Merck \& Co

"http://www.zervita.de“" (Zervita)

Fachgesellschaften/Forschungsinstitute

Deutsches Krebsforschungsinstitut

Gynäkologische und Pädiatrische Fachgesellschaften

Deutsche Gesellschaft für Epidemiologie Deutsches Netzwerk Evidenzbasierte Medizin

Deutsche Gesellschaft für Sozialmedizin und

Prävention

Deutsche Gesellschaft für Medizinische Infor-

matik, Biometrie und Epidemiologie

Krebsfachgesellschaften

Arbeitsgemeinschaft der wissenschaftlichen medizinischen Fachgesellschaften

Beratungseinrichtungen/Patientenportale

Pro Familia

Selbsthilfe Kontakt und Informations Stelle

(SEKIS)

Nationale Kontakt- und Informationsstelle zur Anregung und Unterstützung von Selbsthilfegruppen (NAKOS)

"http://www.patienteninformation.de" von Bundesärztekammer und Kassenärztlicher

Bundesvereinigung

"http://www.akdae.de" der Arzneimittelkommission der deutschen Ärzteschaft, Techniker Krankenkasse und BMG

"http://www.gesundheitsinformation.de" des Instituts für Qualität und Wirtschaftlichkeit im Gesundheitswesen satz erläutert wurde (bei der HPV-Impfung also die Krebsprävention über den Weg des Schutzes vor einer HPV-Infektion). Das vierte Modul „Vor- und Nachteile der Maßnahmen" erfasste die Darstellung von Wirksamkeit und Kosten der HPV-Impfung und des Screenings und bei der HPV-Impfung zusätzlich den Hinweis, dass sie das Pap-Screening nicht ersetzt. Beim Pap-Screening wurden zudem die Kommunikation von Testgütekriterien wie Sensitivität und Spezifität, positiver und negativer prädiktiver Wert und die Konsequenzen falsch-positiver und falsch-negativer Befunde kodiert.

Die Bewertungskriterien, nach denen geprüft wurde, ob die Mindestanforderungen an Informationen für Laien erfüllt waren, umfassten:

1. zur Vollständigkeit: die Angabe von Vor- und Nachteilen der Intervention, ihrer Wirksamkeit, von Nebenwirkungen, Testgüte und falsch-positiven Befunden,

2. zur Transparenz: die Angabe natürlicher Häufigkeiten und/oder die Übersetzung bedingter Wahrscheinlichkeiten in Häufigkeitsbäume,

\section{3. zur Richtigkeit: die Darstellung des}

Bezuges zu Zielgrößen, von Unsicherheiten und Zahlengrößen [7].

Soweit Zahlen berichtet wurden, erlaubten wir Abweichungen von den Daten in den Facts-Boxes: So haben wir zum Beispiel beim Pap-Screening Sensitivitätsangaben von deutlich unter $50 \%$ als richtig akzeptiert, obwohl wir eine sehr optimistische Sensitivität von $70 \%$ für höhergradige Dysplasien zugrunde legen. In Deutschland liegt die Sensitivität oft bei unter $50 \%$; zudem ist sie bei jüngeren Frauen geringer als bei älteren [38, 39, 40]. Entsprechend haben wir bei der
HPV-Impfung Angaben zu Risikoreduktionen von einem Drittel bezogen auf alle Dysplasien als empirischen Nachweis akzeptiert, auch wenn unsere Zahlen niedriger liegen.

Vor der endgültigen Kodierung wurde das Kodierschema in einem Pretest erprobt und modifiziert. Somit sollte eine einheitliche und systematische Bewertung des Materials gewährleistet sein.

Die Inter-Rater-Reliabilität zeigte an einer zufälligen Stichprobe von $\mathbf{2 1} \%$ der Webseiten (13 von 61 Seiten) mit KappaWerten von 0,6 bis 0,7 eine gute Übereinstimmung [41]. Es erfolgte eine deskriptive Auswertung anhand der Module und Medienarten. Die Ethik-Kommission des Max-Planck-Institutes für Bildungsforschung Berlin erteilte vorab ein positives Votum für die Studie.

\section{Ergebnisse}

\section{Suchergebnisse}

Insgesamt wurden 1781 Praxis-Broschüren, Zeitungsartikel und Webseiten zur HPV-Impfung und zum Pap-Screening gefunden. Von diesen wurden $240 \mathrm{Be}$ richte evaluiert. 220 Beiträge berichteten über die Impfung und 20 über das Screening; elf dieser Berichte behandelten beide Themen. Die 220 Materialien zur Impfung umfassten 18 Praxis-Broschüren, 141 Zeitungsartikel und 61 Webseiten, die 20 Materialien zum Pap-Screening vier Praxis-Broschüren, sechs Zeitungsartikel und zehn Webseiten.

Die Nachforschungen in 35 Arztpraxen erbrachten 20 Broschüren; 16 beschäftigten sich mit der HPV-Impfung, zwei mit beiden Themen und zwei ausschließlich mit dem Pap-Screening.

Von ursprünglich 1586 Pressemeldungen blieben aufgrund von Dopplungen $(n=144)$, zu kurzer oder thematisch nicht-relevanter Beiträge (Veranstaltungshinweise, Berichte über Nobelpreisverleihung, Management- und Investmentberichte der Pharmabranche etc., $\mathrm{n}=907$ ), nicht in Deutschland erschienener Berichte $(n=244)$ oder aufgrund unspezifischer Ausschlussgründe (zum Beispiel fehlende Texte in der Trefferliste, nur Überschriften, vor Suchzeitraum veröffentlicht: $n=146) 145$ übrig, die aus- 
gewertet wurden. 139 Zeitungsartikel informierten ausschließlich über die HPVImpfung, zwei über beide Themen sowie vier nur über das Pap-Screening.

Von zunächst 175 potenziell interessanten Webseiten wurden aufgrund der bereits oben genannten Ausschlusskriterien (Dopplungen: $\mathrm{n}=16$, zu kurz oder thematisch nicht relevant: $n=84$, nicht deutsch: $n=4$, wissenschaftlicher Beitrag: $n=3$, unspezifisch: n=4) 64 ausgewertet. Es handelte sich bei ihnen überwiegend um Materialien zur HPV-Impfung. Sieben Internetinformationen thematisierten beide Interventionen und drei nur das PapScreening.

\section{Medienanalyse}

Bei zwei Dritteln der Medienberichte zur HPV-Impfung (146 von 220 Materialien) wurde der Beitragsstil von uns als ausgewogen beziehungsweise aufklärend empfunden (• Tab. 3); 39\% von ihnen (86 von 220 Berichten) wurden als tendenziös im Sinne eines werbenden beziehungsweise überredenden Charakters, aber nur $4 \%$ als warnend eingestuft ( 9 von $220 \mathrm{Be}$ richten). Hierbei waren Mehrfachbewertungen möglich, sodass unterschiedliche Passagen innerhalb eines längeren Textes teilweise unterschiedliche Kodierungen erhielten.

Eine Visualisierung zum besseren Verständnis der Epidemiologie des Gebärmutterhalskrebses und zum Nutzen der HPV-Impfung erfolgte selten. Die Mehrheit der Berichte zur HPV-Impfung richtete sich an die Allgemeinbevölkerung (92\%, 202 von 220 Berichten) oder gezielt an Eltern, Töchter und Angehörige. Soweit Akteure explizit in den Informationen genannt wurden, traten insbesondere Wissenschaftler und Ärzte in den Vordergrund (in $75 \%$ beziehungsweise $57 \%$ der Berichte; 164 beziehungsweise 125 von 220 Berichten), teilweise auch Töchter und Eltern (17\%, 37 von 220 Berichten).

Während alle Materialien zur HPVImpfung auf das Ziel der Krebsprävention hinwiesen, erklärten nur 154 von 220 Berichten (70\%) explizit, dass dieses Ziel indirekt über den Weg des Schutzes vor einer HPV-Infektion durch eine Impfung erreicht werden soll. Die Hälfte machte Angaben zur Morbidität und

\begin{tabular}{|c|c|c|c|c|c|}
\hline Modul & Kategorie & $\begin{array}{l}\text { Alle Ma- } \\
\text { terialien } \\
(\mathrm{N}=220) \\
\%(n)\end{array}$ & $\begin{array}{l}\text { Praxis-Bro- } \\
\text { schüren } \\
(\mathrm{N}=18) \\
\%(n)\end{array}$ & $\begin{array}{l}\text { Zeitungs- } \\
\text { artikel } \\
(\mathrm{N}=141) \\
\%(\mathrm{n})\end{array}$ & $\begin{array}{l}\text { Web- } \\
\text { Seiten } \\
(\mathrm{N}=61) \\
\%(n)\end{array}$ \\
\hline \multirow{14}{*}{$\begin{array}{l}\text { Allgemei- } \\
\text { nes }\end{array}$} & \multicolumn{5}{|l|}{ Art des Beitrags ${ }^{\mathrm{a}}$} \\
\hline & - Hauptartikel & $48(106)$ & $67(12)$ & $47(66)$ & $46(28)$ \\
\hline & - Kurzartikel & $51(112)$ & $33(6)$ & $33(73)$ & $54(33)$ \\
\hline & - Update/Sonstiges & $11(25)$ & $28(5)$ & $9(12)$ & $13(8)$ \\
\hline & \multicolumn{5}{|l|}{ Ton des Beitrags/Beitragsstila } \\
\hline & -Werben/überzeugen/überreden & $39(86)$ & $44(8)$ & $28(39)$ & $6(39)$ \\
\hline & - Ausgewogen/aufklären & $66(146)$ & $78(14)$ & $72(101)$ & $51(31)$ \\
\hline & - Warnen & $4(9)$ & $0(0)$ & $5(7)$ & $3(2)$ \\
\hline & - Sonstiges/nicht zuzuordnen & $3(7)$ & $0(0)$ & $4(5)$ & $3(2)$ \\
\hline & \multicolumn{5}{|l|}{ Visualisierung } \\
\hline & - Epidemiologie & n.a. & $6(1)$ & n.a. & $10(6)$ \\
\hline & - Nutzen/Effektivität & n.a. & $0(0)$ & n.a. & $7(4)$ \\
\hline & $\begin{array}{l}\text { - Biologie/Virus/Gewebeveränderung/ } \\
\text { Anatomie }\end{array}$ & n.a. & $39(7)$ & n.a. & $10(6)$ \\
\hline & - Fotos $^{\mathrm{c}}$ & n.a. & $67(12)$ & n.a. & $20(12)$ \\
\hline \multirow[t]{15}{*}{ Akteure } & \multicolumn{5}{|l|}{ Akteure $\mathrm{a}^{\mathrm{a}}$} \\
\hline & -Wissenschaftler & $75(164)$ & $17(3)$ & $78(110)$ & $84(51)$ \\
\hline & - Ärzte & $57(125)$ & $28(5)$ & $55(77)$ & $70(43)$ \\
\hline & - Patientenvertreter & $9(20)$ & $0(0)$ & $8(11)$ & $15(9)$ \\
\hline & - Eltern/Töchter & $17(37)$ & $0(0)$ & $6(9)$ & $46(28)$ \\
\hline & - Sonstige/unspezifisch & $54(119)$ & $89(16)$ & $51(72)$ & $51(31)$ \\
\hline & \multicolumn{5}{|l|}{ Zielgruppe $e^{\mathrm{a}}$} \\
\hline & - Laien allgemein & $92(202)$ & $44(8)$ & $99(139)$ & $90(55)$ \\
\hline & - Ärzte & $26(58)$ & $6(1)$ & $12(17)$ & $66(40)$ \\
\hline & - Wissenschaftler & $23(50)$ & $0(0)$ & $12(17)$ & $54(33)$ \\
\hline & - Eltern & $78(172)$ & $94(17)$ & $71(100)$ & $90(55)$ \\
\hline & - Angehörige/Peers & $62(136)$ & $50(9)$ & $56(79)$ & $79(48)$ \\
\hline & - Betroffene/Mädchen & $75(165)$ & $94(17)$ & $69(97)$ & $84(51)$ \\
\hline & - Beratungsstellen & $23(50)$ & $0(0)$ & $12(17)$ & $54(33)$ \\
\hline & - Sonstige & $45(100)$ & $6(1)$ & $46(65)$ & $56(34)$ \\
\hline \multirow[t]{6}{*}{$\begin{array}{l}\text { Risiko/ } \\
\text { Ursachen }\end{array}$} & $\begin{array}{l}\text { Impfziel der Krebsprävention über Infek- } \\
\text { tionsschutz }\end{array}$ & $70(154)$ & $33(6)$ & $66(94)$ & $89(54)$ \\
\hline & Epidemiologie/Häufigkeit & $57(125)$ & $50(9)$ & $54(76)$ & $66(40)$ \\
\hline & $\rightarrow$ Korrekte Werte & $46(102)$ & $33(6)$ & $43(61)$ & $57(35)$ \\
\hline & $\rightarrow$ Häufigkeitsvergleiche & $16(36)$ & $6(1)$ & $12(17)$ & $30(18)$ \\
\hline & Krebsursachen $^{b}$ & $45(99)$ & $72(13)$ & $38(54)$ & $52(32)$ \\
\hline & Möglichkeit der Spontanheilung & $23(50)$ & $50(9)$ & $13(18)$ & $38(23)$ \\
\hline \multirow{13}{*}{$\begin{array}{l}\text { Vor- und } \\
\text { Nachteile } \\
\text { der Maß- } \\
\text { nahmen }^{\mathrm{a}}\end{array}$} & Vor- und Nachteile erwähnt & $48(105)$ & $17(3)$ & $50(70)$ & $52(32)$ \\
\hline & \multicolumn{5}{|l|}{ Nutzen/Impfeffektivität } \\
\hline & Irgendwelche Zahlenangaben & $41(90)$ & $17(4)$ & $38(56)$ & $48(30)$ \\
\hline & \multicolumn{5}{|l|}{ RRR } \\
\hline & $\rightarrow$ Dysplasie & $13(29)$ & $11(2)$ & $11(15)$ & $20(12)$ \\
\hline & $\rightarrow$ Korrekte Werte & $6(14)$ & $0(0)$ & $6(8)$ & $10(6)$ \\
\hline & \multicolumn{5}{|l|}{ ARR } \\
\hline & $\rightarrow$ Dysplasie & $2(5)$ & $0(0)$ & $1(2)$ & $5(3)$ \\
\hline & $\rightarrow$ Korrekte Werte & $0(0)$ & $0(0)$ & $0(0)$ & $0(0)$ \\
\hline & Angabe Präventionspotenzial & $0(1)$ & $0(0)$ & $0(0)$ & $2(1)$ \\
\hline & - Korrekte Werte & $0(1)$ & $0(0)$ & $0(0)$ & $2(1)$ \\
\hline & Wirksamkeit auf Krebs unklar & $6(14)$ & $0(0)$ & $4(6)$ & $13(8)$ \\
\hline & Nebenwirkungen & $25(56)$ & $39(7)$ & $14(20)$ & $48(29)$ \\
\hline
\end{tabular}


Tab. 3 Medienanalyse der Informationen zur HPV-Impfung (Fortsetzung)

\begin{tabular}{|c|c|c|c|c|c|}
\hline Modul & Kategorie & $\begin{array}{l}\text { Alle Ma- } \\
\text { terialien } \\
(\mathrm{N}=220) \\
\%(n)\end{array}$ & $\begin{array}{l}\text { Praxis-Bro- } \\
\text { schüren } \\
(\mathrm{N}=18) \\
\% \text { (n) }\end{array}$ & $\begin{array}{l}\text { Zeitungs- } \\
\text { artikel } \\
(\mathrm{N}=141) \\
\%(\mathrm{n})\end{array}$ & $\begin{array}{l}\text { Web- } \\
\text { Seiten } \\
(\mathrm{N}=61) \\
\%(\mathrm{n})\end{array}$ \\
\hline & -Zahlenangaben & $15(32)$ & $22(4)$ & $7(10)$ & $30(18)$ \\
\hline & Explizite Impfempfehlung & $40(89)$ & $44(8)$ & $29(41)$ & $66(40)$ \\
\hline & Hinweis aufPap-Test & $47(104)$ & $89(16)$ & $36(51)$ & $61(37)$ \\
\hline & Kosten & $37(81)$ & $11(2)$ & $44(62)$ & $28(17)$ \\
\hline & - Pro Injektion & $5(12)$ & $0(0)$ & $4(6)$ & $10(6)$ \\
\hline & - Pro Impfung & $32(70)$ & $6(1)$ & $39(55)$ & $23(14)$ \\
\hline & - Pro Jahrgang & $5(10)$ & $0(0)$ & $5(7)$ & $5(3)$ \\
\hline & - Kostenvergleich & $6(13)$ & $0(0)$ & $6(8)$ & $8(5)$ \\
\hline
\end{tabular}

ARR absolute Risikoreduktion, n.a. nicht anwendbar (LexisNexis stellt in der Regel nur Texte zur Verfügung), RRR

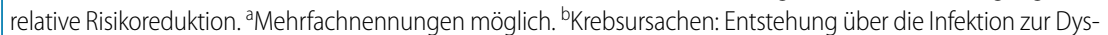
plasie bis hin zum Krebs innerhalb langer Zeiträume. 'Fotos beinhalten gesunde, junge, glückliche Frauen, Mütter und Töchter sowie einen Vater oder ein Liebespaar und einmal einen Arzt mit Spritze.

Tab. 4 Medienanalyse der Informationen zum Pap-Screening

\begin{tabular}{|ll|}
\hline Modul/Kategorie & $\begin{array}{l}\text { Materialien zum } \\
\text { Thema Pap- } \\
\text { Screening (N=20) } \\
\%(\mathbf{n})\end{array}$ \\
\hline Testgenauigkeit & \\
\hline Sensitivität & $15(3)$ \\
$\rightarrow$ Korrekte Zahlen & $15(3)$ \\
\hline Spezifität & $5(1)$ \\
\hline$\rightarrow$ Korrekte Zahlen & $5(1)$ \\
\hline PPV & $0(0)$ \\
\hline$\rightarrow$ Korrekte Zahlen & $0(0)$ \\
\hline NPV & $0(0)$ \\
\hline$\rightarrow$ Korrekte Zahlen & $0(0)$ \\
\hline Hinweis auf Falsch-Positive & $25(5)$ \\
\hline Darstellungsformat & \\
\hline$\rightarrow$ Prozent & $20(4)$ \\
\hline$\rightarrow$ Absolute Zahlen & $5(1)$ \\
\hline$\rightarrow$ Häufigkeitsbaum & $0(0)$ \\
\hline Nutzen & \\
\hline Präventionspotenzial & $25(5)$ \\
\hline$\rightarrow$ Korrekte Werte & $25(5)$ \\
\hline$\rightarrow$ Absolute Zahlen & $25(5)$ \\
\hline $\begin{array}{l}\text { PPV/NPV Positiv-/negativ-prädiktiverWert. } \\
\text { aMehrfachnennungen möglich. }\end{array}$ \\
\hline
\end{tabular}

Mortalität des Gebärmutterhalskrebses (57\%, 125 von 220 Berichten). Die hier genannten Zahlen entsprachen in $46 \%$ der Berichte (102 von 220 Berichten) den von uns ermittelten Werten. Auf Häufigkeitsvergleiche zur Einordnung des Grundrisikos, an einem Zervixkarzinom zu erkranken, wurde meist verzichtet (in $84 \%$ der Berichte, 184 von 220 Berichten).

Der Anteil der Berichte, die Vor- und Nachteile der HPV-Impfung erwähnten, belief sich auf $48 \%$ (105 von 220 Berichten). Zahlenangaben zur Effektivität der Impfung fanden sich in nur $41 \%$ (9o von 220 Berichten) der Materialien, 13\% (29 von 220 Berichten) bezogen sich hierbei auf die relative Risikoreduktion bezüglich des maßgeblichen Surrogatparameters aller zervikalen Dysplasien, und lediglich 6\% (14 von 220 Berichten) umfassten die vorab festgelegten Inhalte der Facts-Box. Das transparente Maß der absoluten Risikoreduktion für das Auftreten von Dysplasien wurde in nur fünf Beiträgen verwendet, jedoch war hier keine Zahlenangabe korrekt. Das Präventionspotenzial wurde einmal richtig präsentiert. 6\% der Materialen (14 von 220 Beiträgen) gaben an, dass die Wirksamkeit der Impfung auf das Zervixkarzinom als direkt gemessene Zielgröße bislang nicht untersucht ist. Im Zusammenhang mit der Wirksamkeit wurden die Zahlen 70 bis $80 \%$ in 43 (20\%) und höhere Zahlen von über 80 bis $100 \%$ in 34 der 220 Beiträge (15\%) genannt - und zwar bezogen auf ein Durcheinander von (a) dem Zervixkarzinomanteil, der HPV 16/18 zuzuschreiben ist, (b) Infektionen, Krebsvorstufen, Krebserkrankungen, Sterblichkeit oder (c) völlig ohne Bezug zu einem bestimmten Outcome. Unter den Beiträgen mit den Angaben von 70 bis $80 \%$ stellten nur sechs (3\%) klar, dass es sich um einen ätiologischen und nicht um einen Wirksamkeitsnachweis handelt.

Die Evaluation der Angaben zu Impfnebenwirkungen ergab, dass nur ein Viertel der identifizierten Berichte (56 von 220 Beiträgen) diese diskutierte; dabei fanden sich in lediglich 15\% (32 von 220 Beiträgen) korrekte Zahlen zur Häufigkeit ihres Auftretens. In 40\% der Beiträge (89 von 220 Beiträgen) wurde eine explizite Impfempfehlung ausgesprochen. Ein Hinweis auf den Pap-Test, den die Impfung nicht ersetzen kann, fand sich in knapp der Hälfte der Materialien (47\%, 104 von 220 Beiträgen). Die Impfkosten wurden in $37 \%$ der Informationen (in 81 von 220 Beiträgen) erwähnt, aber in nur 6\% (13 von 220 Beiträgen) im Zusammenhang mit einem Vergleich zu allgemein bekannten Kostengrößen.

Bewertet man die Beiträge nach der Art des Mediums, zeigt sich, dass nur $33 \%$ der Praxis-Broschüren korrekte epidemiologische Zahlen berichten, während dies bei $43 \%$ der Zeitungsartikel und $57 \%$ der Webseiten der Fall war. In keiner Praxis-Broschüre fanden sich korrekte Angaben zur relativen und absoluten Risikoreduktion. Auch wurde eine Diskussion der Vor- und Nachteile der HPV-Impfung vergleichsweise selten vorgenommen ( $17 \%$ versus $50 \%$ versus $52 \%$ ). Der werbende Charakter stand bei den Praxis-Broschüren stärker als bei den anderen Medien im Vordergrund (44\% versus $28 \%$ versus $6 \%$ ).

Mit Blick auf das Pap-Screening machten acht von 20 Materialen (40\%) qualitative und/oder quantitative Angaben zur Testgüte; vier nannten konkrete Zahlen $(20 \%)$, von diesen enthielt ein Beitrag absolute Zahlenangaben (• Tab.4). Keiner nutzte die leicht verständliche Darstellung der Testgüte in einem Häufigkeitsbaum. Fünf Materialien gaben Hinweise auf die Möglichkeit falsch-positiver Befunde (25\%). Alle Beiträge gingen von einer Wirksamkeit des Screenings zur Krebsprävention aus. Sechzehn machten qualitative oder quantitative Aussagen über seine Effektivität (8o\%). Fünf präsentierten Zahlen zum Präventionspotenzial (25\%). Insgesamt lässt sich feststellen, dass die genannten Zahlen - soweit sie überhaupt berichtet wurden korrekt waren. Die Hälfte der Beiträge hatte einen werbenden Stil (zehn von 20 Beiträgen), 65\% erschienen ausgewogen (13 von 20 Beiträgen) und einer warnend; ein Beitrag war diesen Kategorien nicht zuzuordnen. 
Die Mindestanforderungen an eine ausgewogene Information für Laien erfüllte einer von 220 Beiträgen zur HPVImpfung beziehungsweise einer von 20 Beiträgen zum Pap-Screening.

\section{Diskussion}

In der vorliegenden Medienanalyse zur Zervixkarzinomprävention in Deutschland wurden 220 Materialien zur HPVImpfung mit Gardasil ${ }^{\circledR}$ und 20 zum PapScreening untersucht.

Obwohl alle Beiträge zur HPV-Impfung ihre präventive Wirkung anführten, enthielten nur $41 \%$ konkrete Zahlenangaben über ihre Effektivität (9o von 220 Beiträgen). In $7 \%$ der Beiträge ( 15 von 220 Beiträgen) waren die Angaben korrekt; 48\% (105 von 220 Beiträgen) erwähnten die Vor- und Nachteile der Impfung. Nur $2 \%$ der Beiträge ( 5 von 220 Beiträgen) verwendete absolute Zahlenangaben zur Beschreibung der Risikoreduktion für das Auftreten einer Dysplasie; jedoch war keine richtig. Lediglich in einem Beitrag fanden sich korrekte Angaben zum Präventionspotenzial. Zahlenangaben zum Nutzen der Impfung waren insbesondere in Praxisbroschüren selten vorhanden. Dies ist umso bedenklicher, als mit ihnen weniger die Allgemeinheit, sondern gezielt Betroffene zur konkreten Entscheidungsfindung angesprochen werden sollen. Dabei fällt auf, dass bei ihnen - verglichen mit anderen Medien - eher der werbende als der ausbalancierte Charakter im Vordergrund stand. Besonders selten wurden in den Praxisbroschüren die Vor- und Nachteile der Impfung diskutiert. Diese Defizite sind umso gravierender als sie den größten Seitenumfang aufwiesen. Auf das Pap-Screening wurde in ihnen jedoch häufig hingewiesen - vermutlich um beide Interventionen leichter zu vermarkten. Diese Aussagen zur Qualität der unterschiedlichen Medienarten (Praxisbroschüren versus Zeitungsartikel versus Webseiten) sind aber vorsichtig zu bewerten, da in der Studie eine vergleichsweise kleine Auswahl an Praxisbroschüren untersucht wurde.

Demgegenüber sind die untersuchten Zeitungsartikel, die aus der umfassenden LexisNexis-Datenbank aller deutschen Pressemeldungen ermittelt wurden, eine besonders repräsentative Stichprobe. In Bezug auf die Auswahl an Webseiten war die Internetrecherche zwar limitiert, bildet aber einschlägige Public-Health-Institutionen in Deutschland ab. Im Gegensatz dazu entstammen die Praxisbroschüren nur zwei Berliner Bezirken; dafür bilden sie aber soziodemografisch divergent alle Kinder- und Frauenarztpraxen dieser Bezirke ab, sodass es sich bei ihnen insgesamt um eine aussagefähige Stichprobe handelt. Potenzielle Selektionsverzerrungen halten sich somit in Grenzen. Obwohl die Kodierungen aufgrund der Heterogenität des Datenmaterials variieren, halten sich aufgrund der Durchführung des Pretests potenzielle Klassifikationsfehler - wie die Kappa-Werte zeigen - ebenfalls in Grenzen, zumal nicht nur dichotome, sondern auch narrative Kodierungen einflossen. Dem möglichen Einwand einer zu rigide gefassten Impfeffektivität ist zu entgegnen, dass sich das Ergebnis einer diffusen Berichterstattung selbst bei einer optimistischen Effektivitätsannahme von bis zu 70\% Krebsreduktion [42] nicht verändert, da auch diese Aussage selten richtig antizipiert wird. Das Problem liegt weniger in Zahlengrenzen als im prinzipiellen Missverständnis wissenschaftlicher Erkenntnisse.

In nur vier von 20 Berichten zum PapScreening (20\%) fanden sich Zahlenangaben zur Testgüte, einer von diesen enthielt absolute Zahlenangaben. Fünf Berichte wiesen auf die Möglichkeit falschpositiver Befunde hin (25\%). Fünf machten Angaben zum extrapolierten Präventionspotenzial. Ähnliche Ergebnisse finden sich für deutsche Aufklärungsbroschüren zum Mammographie-Screening: Demnach enthalten bis zu 19\% Angaben zur Testgüte, 11\% Angaben zu den Konsequenzen falsch-positiver Befunde und 7\% Nutzenangaben, das heißt Angaben zur absoluten und relativen Risikoreduktion [43]. Diese Informationsdefizite sind allerdings nicht nur ein deutsches, sondern auch ein internationales Phänomen [7].

Obwohl das Pap-Screening seit Jahrzehnten etabliert ist, werden erstaunlich wenige Zahlen zur Testgüte und Wirksamkeit berichtet, auch wenn die Literatur dazu weit eindeutiger Auskunft gibt als bei der Impfung. Die Eindeutigkeit zeigt sich auch daran, dass die Zahlen - wenn sie berichtet werden - korrekt sind. Insgesamt wurden weit weniger Berichte zum Pap-Screening als zur HPV-Impfung gefunden - vermutlich deshalb, weil es bereits seit Jahrzehnten implementiert ist und folglich keinen Neuigkeitswert aufweist. Da die HPV-Impfung das PapScreening nicht ersetzen kann, wäre es sinnvoll, dies in den Berichten zu erwähnen, um die Teilnahmerate am Screening - infolge eines unzutreffenden Sicherheitsgefühls nach einer Impfung - nicht zu verringern. Trotz des diesbezüglich bestehenden wissenschaftlichen Konsenses weist nicht einmal die Hälfte der Materialien zur HPV-Impfung darauf hin. Die Mindestanforderung an die Vollständigkeit (Darlegung von Vor- und Nachteilen), die Transparenz (Angabe von absoluten Zahlen) und die Richtigkeit der Informationen erfüllten zur HPV-Impfung einer von 220 und zum Pap-Screening einer von 20 Berichten.

Ungeachtet umfangreicher Forschungsergebnisse zur Risikokommunikation, wissenschaftliche Erkenntnisse allgemein verständlich darzulegen $[1,2,3$, $5,7,43,44]$, zeigt diese Medienanalyse einmal mehr, dass Transparenz nach wie vor die Ausnahme ist: Ein Großteil der Medienberichte zur HPV-Impfung und zum Pap-Screening ist einseitig und überhöht den Nutzen, sodass eine ausgewogene Darstellung der Vor- und Nachteile der beiden Maßnahmen oftmals nicht vorhanden ist. Die Angabe absoluter Zahlen ist so unterrepräsentiert wie die Intransparenz relativer Maße überwiegt - sofern überhaupt Zahlen genannt werden. Einseitige Wahrnehmungen finden sich allerdings nicht nur in den Medien, sondern auch in der Allgemeinbevölkerung - beispielsweise zum Mammographie- und PSA-Screening [45].

Interessant ist der Befund, dass die Mehrheit der Berichte zur HPV-Impfung und zum Pap-Screening zunächst aufklärend und ausgewogen klingt (66\% und 65\%). Bei näherem Hinsehen fehlen ihnen aber essenzielle Daten, um dem Laien eine informierte Abwägung zu ermöglichen. Dieser Befund könnte Ausdruck einer zunehmenden öffentlichen Diskussion zur Risikokommunikation sein, in der zwar eine Sensibilisierung für die Notwendigkeit ausbalancierter Infor- 


\section{Originalien und Übersichten}

Tab. 5 Mindeststandards für medizinische Informationen in der Laienkommunikation

1. Wissenschaftliche Diskussionsebenen

- Grundsätzlich sind Plausibilitätsbetrachtungen, Expertenmeinungen, Theorien und Hypothesen von empirischen Überprüfungen zu differenzieren, da Letztere höhere Evidenzlevel bekleiden

- Darüber hinaus sind empirische Nachweise zur Ätiologie einer Erkrankung von den empirischen Nachweisen zur Wirksamkeit der Interventionen abzugrenzen, da empirische Erkenntnisse zu den Ursachen einer Erkrankung nicht gleichbedeutend mit Wirksamkeitsnachweisen zu ihrer Bekämpfung sind

2. Differenzierung verschiedener Formen von medizinischen Maßnahmen

- Screenings oder diagnostische Tests unterscheiden sich von primärpräventiven oder therapeutischen Interventionen, da hier verschiedene Bewertungskriterien von Relevanz sind. Der potenzielle Nutzen und Schaden wird bei primärpräventiven Interventionen unmittelbar durch Morbiditäts-/Mortalitätsveränderungen abgebildet, während beim Screening zwei Bewertungsschritte erfolgen, die die Testgenauigkeit mit ihren Folgen und auch die Wirkung der Früherkennung auf die Krankheitslast erfassen

\section{Darstellung von Nutzen und Schaden}

- Für informierte Entscheidungen ist die Darstellung des potenziellen Nutzens und Schadens einer Intervention, von Unsicherheiten und etwaigen Erkenntnislücken essenziell; Angaben zum Nutzen sollten zudem die Beschreibung von Population und Zeitraum, auf die sich die Erkenntnis bezieht, sowie den expliziten Bezug zum Outcome als indirekt (Surrogat) oder direkt gemessene Zielgröße enthalten, da Surrogatparameter (zum Beispiel Krebsvorstufen) aufgrund ihrer Stellvertreterfunktion für das Krankheitsgeschehen größere Unsicherheiten als direkt gemessene Zielgrößen (zum Beispiel die Krebsendstufe) implizieren

- Prinzipiell sind absolute Zahlen mit der Angabe von Basisraten gegenüber relativen Zahlen vorzuziehen und mit Vergleichszahlen bekannter Krankheitsentitäten und Ausgabenbudgets zu versehen, um die Zahlen einordnen zu können. Zur Darstellung der Testgenauigkeit ist die Verwendung von Zahlenbäumen mit natürlichen Häufigkeiten erforderlich

- Angaben zum extrapolierten Präventionspotenzial und zu den Kosten sind erforderlich, um verständlich zu machen, wie begrenzte öffentliche Ressourcen investiert werden

\section{Wertung von Informationen}

- Grundsätzlich wäre es sinnvoll, den Unterschied zwischen Informationen und deren Wertung zu antizipieren, da der Wertung oftmals auf Kosten essenzieller Informationen überproportionale Gewichtung zukommt. Obwohl starke Bewertungen spannender und infolgedessen besser verkäuflich erscheinen, tragen ausgewogene Informationen zur Vertrauensbildung und Nachhaltigkeit bei

mationen stattgefunden hat, diese aber faktisch nach wie vor nicht hinreichend mit entsprechenden Zahlen unterlegt werden. Der Anteil rein werbender Berichterstattung ist in den Materialien zur HPV-Impfung mit 39\% und in den Materialien zum Pap-Screening mit 50\% immer noch hoch.

Ein Verständnisproblem bei der Berichterstattung zur Effektivität der HPVImpfung betrifft die ätiologische Erkenntnis, dass 70\% der Gebärmutterhalskrebse mit HPV 16/18 und 20\% mit anderen onkogenen HPV-Typen assoziiert sind [46]. Da also HPV 16/18 die häufigsten Verursacher des Gebärmutterhalskrebses sind, wurde zuerst ein Impfstoff gegen diese Virustypen entwickelt. Das ist ausgesprochen plausibel. Das heißt jedoch nicht, dass durch die Impfung 70\% der Zervixkarzinome „verschwinden“, was aber nicht selten in den Berichten behauptet wird. Auch ist ein nahezu 100\%iger Schutz vor einer HPV-16/18-Infektion nicht mit einem 100\%igen Schutz vor einer Zervixkarzinom-Erkrankung gleichzusetzen. Um die tatsächlichen Auswirkungen der Impfung auf das Krankheitsgeschehen zu prüfen, müssen klinische Studien durchgeführt werden: Beispielsweise könnte ein HPV-16/
18-Replacement durch andere Virustypen stattfinden, es können Kreuzimmunitäten auftreten, und die Impfwirkung kann bei vorab nicht mit HPV 16/18 infizierten Frauen anders ausfallen als bei vorab infizierten. Darüber hinaus sind Fragen zur Dauer der Immunität und zur Möglichkeit einer Boosterung durch Nachimpfen offen. Diese Fragen lassen sich ex ante, das heißt ohne empirische Untersuchung zur Wirksamkeit der Impfung, nicht beantworten. So ist zwischen den ätiologischen Erkenntnissen zu den Risikofaktoren für die Ausprägung einer Krebserkrankung (70\% aller Zervixkarzinome sind mit HPV 16/18 assoziiert), daraus abgeleiteten Hypothesen zur Entwicklung von Innovationen (70\% der Karzinome könnten durch die Impfung verhindert werden) und der Wirksamkeit der Präventionsmaßnahme $(13,5$ bis $27 \%$ aller Krebsvorstufen wurden bei den Studienteilnehmerinnen in einem Nachbeobachtungszeitraum von drei Jahren durch die Impfung verhindert [21]) zu differenzieren. Ergebnisse aus Studien haben zudem Auswirkung auf die konkrete Ausgestaltung der Impfempfehlung. So wird die Impfung für Nicht-Infizierte vor dem ersten Geschlechtsverkehr empfohlen, da sie sich dann am wirksamsten zeigte.
Die besagte Zahl von 70\% wird in den Informationsmaterialien bezogen auf die Reduktion der Erkrankungs- als auch Sterblichkeitsrate genannt. Die ätiologischen Erkenntnisse besagen aber lediglich - wie oben bereits ausgeführt -, dass $70 \%$ der Gebärmutterhalskrebse mit HPV 16/18 assoziiert sind. Auch werden viele Prozentzahlen oftmals ohne Bezug zur Population, Basisrate, zu Zielgrößen (Infektion, Dysplasie, Krebs) und zum Untersuchungszeitraum angeführt. Solche Aussagen haben keinen Informationsgehalt und suggerieren eine vermeintliche Sicherheit, insbesondere dann, wenn von einem über 90\%- oder 100\%igen Schutz vor einer Krebserkrankung die Rede ist, wobei sich Letzterer aber tatsächlich nur auf die Infektion mit HPV 16/18 oder auf HPV-16/18-assoziierte Dysplasien bei HPV-16/18-naiven Frauen beziehen kann. Verfügbare empirische Daten zur Wirksamkeit der Impfung betreffen also nur die Immunität gegen HPV 16/18 und den Krebssurrogatmarker „Dysplasie“. Die heute vorliegenden Daten ermöglichen aber noch keine Aussagen über die unmittelbare Auswirkung der Impfung auf die eigentliche Zielkrankheit, das heißt auf den eigentlichen Endpunkt „Zervixkarzinom“, da die Gewinnung diesbezüg- 
licher Daten jahrzehntelange Beobachtungszeiten erfordert, die bislang noch nicht gegeben sind.

Zugegebenermaßen ist die HPV-Impfung aufgrund der Vielzahl unterschiedlicher Studiendaten ein kompliziertes Beispiel; und es gab nicht nur in den Medien, sondern auch in der Wissenschaft Kontroversen über ihre Effekte [24, 47, 48]. Umso bedeutender ist die transparente Kommunikation dieser Unsicherheiten. So arbeitet die Cochrane Collaboration oder die STROBE-Initiative kontinuierlich an verbindlichen Standards für eine transparente wissenschaftliche Berichterstattung über Studien [11, 49]. Obwohl Standards keine Garanten für Ausgewogenheit sind, helfen sie zumindest, wesentliche Aspekte systematisch zu erfragen. Demnach sind transparente Maßstäbe in der Wissenschaftskommunikation etablierter als in der Laienkommunikation. Wünschenswert wäre ihre Transformation in laienverständliche Standards, deren Grundlagen in $\bullet$ Tab. 5 zusammengefasst und zukünftig weiter zu differenzieren sind. Sie ergänzen internationale Empfehlungen für Patienteninformationen $[50,51]$, die informierte Entscheidungen ermöglichen [52].

Bei diesen Ergebnissen stellt sich die Frage, was das Ziel von Gesundheitsinformationen sein sollte: Direktiven oder Partizipation? Auch wenn sich Public-HealthAkteure wünschen, Präventionsziele zu erreichen, so darf dabei die Selbstbestimmung des Einzelnen nicht vergessen werden. Hierzu gehört es auch zu tolerieren, dass sich Menschen gegen Empfehlungen entscheiden. Public-Health- und Individualentscheidungen sind von unterschiedlicher Natur. Während Erstere utilitaristischen Prinzipien populationsbezogener Aussagen folgen, handelt es sich bei den Zweiten um Präferenzen Einzelner. So ist es zum Beispiel möglich, dass sich ein Individuum persönlich gegen eine Empfehlung entscheidet, die Public-HealthEntscheidung für den flächendeckenden Einsatz einer Maßnahme aber mit trägt oder umgekehrt. Dieses Phänomen wird beim Pap-Screening deutlich: Durch das Screening lässt sich in Deutschland jährlich eine erhebliche Zahl von Zervixkarzinom-Erkrankungen (10.40o) und Todesfällen (1500) verhindern, dennoch ist die
Wahrscheinlichkeit für die einzelne Frau, an einem solchen Karzinom zu erkranken oder sogar zu versterben gering ( 15 beziehungsweise 3 von 100.00o Frauen jährlich) $[20,32,33]$. Der Einsatz des Screenings ist also aus Populationssicht unmittelbar verständlich, während die Teilnahme der einzelnen Frau zurückhaltender ausfallen kann. Demzufolge gilt die Forderung nach Partizipation auf der Individual- und Populationsebene gleichermaßen, sie führt aber nicht zwangsläufig zu gleichen Entscheidungen.

Obwohl respektiert werden muss, dass ein Teil der Patienten Entscheidungen delegieren möchte und direktive Gesundheitsinformationen wünscht [53], rechtfertigt dies nicht, auf eine ausgewogene Informationsvermittlung zu verzichten. Selbstverständlich steht es auch jedem frei, Empfehlungen abzugeben. Diese sollten mit klaren Argumenten gestützt sein. Da Transparenz in verschiedenen Branchen keinen Marktnachteil bedeutet, sondern vielmehr die Glaubwürdigkeit und das Verbrauchervertrauen erhöht, wäre es unverständlich, sie nicht umzusetzen. Das Eingeständnis von Unsicherheiten ist Voraussetzung für eine informierte Partizipation und die Identifikation mit Interventionsprogrammen, was die Teilnahmerate und Compliance erhöhen kann [54], um auf diese Weise dem gewünschten Gesundheitsziel näher zu kommen als durch selektive Information.

Für die Zukunft wäre es wünschenswert, verbindliche Standards zur Risikokommunikation zu etablieren, deren sich staatliche und gemeinnützige Einrichtungen, Journalisten und Laien gleichermaßen bedienen können. Darüber hinaus wären weitere Medienanalysen zu verschiedenen Gesundheitsthemen und internationale Vergleiche sinnvoll, um die aktuelle Praxis der Risikokommunikation zu prüfen, Lösungen für mögliche Defizite aufzuzeigen und das Ziel der transparenten, ausgewogenen Berichterstattung als unverzichtbare Grundlage für informierte Entscheidungen zu erreichen.

\section{Korrespondenzadresse \\ Dr. A. Neumeyer-Gromen \\ Max-Planck-Institut für Bildungsforschung, \\ Harding Center for Risk Literacy \\ Lentzeallee 94, 14195 Berlin \\ angela_neumeyer@yahoo.de}

\section{N. Bodemer}

Max-Planck-Institut für Bildungsforschung,

Harding Center for Risk Literacy

Lentzeallee 94, 14195 Berlin

bodemer@mpib-berlin.mpg.de

Danksagung. Wir möchten uns bei Frau Claire-Isabel Kellermann, Berlin, für ihre Mitarbeit als studentische Mitarbeiterin in diesem Projekt herzlich bedanken. Sie trug die Medienbeiträge und Informationsmaterialien, die von den verschiedenen Gesundheitseinrichtungen im Internet und in den Arztpraxen öffentlich zugänglich waren, zusammen und unterstützte uns bei der Kodierung des Medienmaterials. Wir danken Herrn Prof. Dr. Ansgar Gerhardus, M.A., MPH, Bremen, für die kritische Durchsicht des Manuskripts.

Interessenkonflikt. Die korrespondierenden Autoren geben an, dass keine Interessenkonflikte bestehen.

\section{Literatur}

1. Cassells A, Hughs M, Cole C et al (2003) Drugs in the news: an analysis of Canadian newspaper coverage of new prescription drugs. CMAJ 168:11331137

2. Moynihan R, Bero L, Ross-Degnan D et al (2000) Coverage by the news media of the benefits and risks of medications. N Engl J Med 342:1645-1650

3. Schwartz LM, Woloshin S, Welch HG (1999) Misunderstandings about the effects of race and sex on physicians' referrals for cardiac catheterization. $\mathrm{N}$ Engl J Med 341:279-283

4. Steckelberg A, Balgenorth A, Mühlhauser I (2001) Analyse von deutschsprachigen Verbraucher-Informationsbroschüren zum Screening auf kolorektales Karzinom. Z Evid Fortbild Qual Gesundhwes 95:535-538

5. Woloshin S, Schwartz LM (2006) Media reporting of research presented at scientific meetings: more caution needed. Med J 184:576-580

6. Gigerenzer G (2002) Reckoning with risk: Learning to live with uncertainty. Penguin Books, London

7. Gigerenzer G, Gaissmaier W, Kurz-Milcke E et al (2007) Helping doctors and patients make sense of health statistics. Psychol Sci Public Interest 2:5395

8. Neumeyer-Gromen A, Bräunlich A, Zeeb H, Razum $O$ (2006) Theorie und Praxis der Epidemiologie Teil I: Systematik theoretischer Grundlagen der Epidemiologie als zentrale Fachdisziplin von Public Health. Prävention Gesundheitsförderung 3:190197

9. Gøtzsche PC, Nielsen M (2009) Screening for breast cancer with mammography. Cochrane Database Syst Rev 4:CD001877

10. Neumeyer-Gromen A, Gaissmaier W (2009) Screening Programs. In: Kattan MW (Hrsg) Encyclopedia of medical decision making. Sage, Cleveland Ohio 
11. Higgins JPT, Green S (Hrsg) (2008) Cochrane handbook for systematic reviews of interventions version 5.0.1 [updated September 2008]. The Cochrane Collaboration. http://www.cochrane.org/resources/handbook/index.htm oder http://www.cochrane-handbook.org

12. Schwartz LM, Woloshin S, Welch HG (2007) The drug facts box: providing consumers with simple tabular data on drug benefit and harm. Med Decis Making 27:655-662

13. Wentzensen N, Klug SJ (2009) Früherkennung des Zervixkarzinoms: Suche nach einem Gesamtkonzept. Dtsch Arztebl 105:617-622

14. Munoz N, Bosch X, de Sanjose S et al (2003) Epidemiologic classification of human papillomavirus types associated with cervical cancer. N Engl J Med 348(6):518-527

15. Schiffman M (2007) Integration of human papillomavirus vaccination, cytology, and human papillomavirus testing. Cancer 111:145-153

16. Ostor AG (1993) Natural history of cervical intraepithelial neoplasia: a critical review. Int J Gynecol Pathol 12:186-192

17. Mühlhauser I, Filz M (2008) Screening auf Zervixkarzinom. a-t 39:29-38

18. Winer RL, Hughes JP, Feng Q et al (2006) Condom use and the risk of genital human papillomavirus infection in young women. $\mathrm{N}$ Engl J Med 354(25):2645-2654

19. Robert Koch-Institut/ RKI (2007) Mitteilung der Ständigen Impfkommission/ STIKO am RKI (Stand: März 2007). Epidemiol Bull 12:97-103

20. Statistisches Bundesamt (2009) Gesundheitsberichterstattung des Bundes (2009). http://www. destatis.de

21. FUTURE II Study Grp (2007) Quadrivalent vaccine against human papillomavirus to prevent highgrade cervical lesions. N Engl J Med 356:19151927

22. FUTURE I investigators (2007) Quadrivalent vaccine against human papillomavirus to prevent anogenital diseases. N Engl J Med 356:1928-1943

23. European Medicine Agency/ EMA (2008) Europ. Beurteilungsbericht (EPAR) GARDASIL (31/10/2008 Gardasil-H-C-703- II-13). http://www.ema.europa.eu/ema/index.jsp?curl=pages/medicines/human/medicines/000703/human_med_000805.jsp \&mid=WC0b01ac058001d125\&murl=menus/medicines/medicines.jsp und http://www.ema.europa.eu/docs/en_GB/document_library/EPAR_Procedural_steps_taken_and_scientific_information after authorisation/human/000703/ WC500021147.pdf

24. Löwer J, Stöcker S (2009) Paul-Ehrlich-Institut: Wie wirksam ist die HPV-Impfung? Dtsch Arztebl 106:386-388

25. Centers for Disease Control and Prevention (CDC) (2008) Vaccine Adverse Event Reporting System (VAERS) 2008. http://www.vaers.hhs.gov/scripts/ data.cfm

26. a-t (Arznei-Telegramm) (2008) 1997 und 2007 im Vergleich - die Umsatzstärksten Arzneimittel. a-t 39:65-66

27. Rosenbrock R (2007) HPV-Impfung - Durchbruch in der Krebsprävention? http://www.forum-gesundheitspolitik.de/artikel/artikel.pl?artikel $=0644$ oder http://www.forum-gesundheitspolitik.de/ dossier/PDF/Rosenbrock-HPV-Impfung.pdf

28. Voß KD (2007) Prävention als Aufgabe der Gesundheitspolitik - Anforderungen an die Gesetzliche Krankenversicherung. http://www.gesundheitberlin.de/download/Vo \%DF_2.pdf
29. Zechmeister I, Freiesleben de Blasio B, Radlberger P et al (2007) Ökonomische Evaluation der Impfung gegen humane Papillomaviren (HPV-Impfung) in Österreich. HTA-Projektbericht Nr. 009, Wien 2007. http://eprints.hta.lbg.ac.at/760/2/HTAProjektbericht_009.pdf

30. European Medicine Agency/ EMA (2010) Gardasil: EPAR - Summary for the public (14/09/2010) http://www.ema.europa.eu/docs/en_GB/document_library/EPAR_-_Summary_for_the_public/human/000703/WC500021146.pdf und http:// www.ema.europa.eu/docs/en_GB/document_library/EPAR_-_Procedural_steps_taken_and scientific_information_after_authorisation/human/000703/WC500021147.pdf

31. Robert Koch-Institut/ RKI (2010) Empfehlungen der Ständigen Impfkommission/ STIKO am RKI/ Stand Juli 2010. Epidemiol Bull 30:235-250

32. Siebert U, Muth C, Sroczynski G et al (2003) Dünnschichtpräparationen und computergestützte Untersuchungen von Zervixabstrichen. Asgard, St. Augustin. http://gripsdb.dimdi.de/de/hta/hta_berichte/hta067_bericht_de.pdf

33. Siebert U, Sroczynski G, Hillemanns P et al (2006) The German Cervical Cancer Screening Model: development and validation of a decision-analytic model for cervical cancer screening in Germany. Eur J Public Health 16:185-192

34. Coste J, Cochand-Priollet B, Cremoux P de (2003) Cross sectional study of conventional cervical smear, monolayer cytology, and human papillomavirus DNA testing for cervical cancer screening. BMJ 7392:733A-736A

35. Petry KU, Breugelmans JG, Benard S et al (2008) Cost of screening and treatment of cervical dyscaryosis in Germany. Eur J Gynaecol Oncol 29:345349

36. Schneider A, Schwarz TF, Hammerschmidt T et al (2007) Treatment patterns and costs of unclear and abnormal cervical conditions in Germany. Geburtsh Frauenheilkd 67:859-865

37. Merten K (1995) Inhaltsanalyse. Einführung in Theorie, Methode und Praxis. Westdeutscher Verlag, Opladen

38. Cuzick J, Clavel C, Petry KU et al (2006) Overview of the European and North American studies on HPV testing in primary cervical cancer screening. Int J Cancer 119:1095-1101

39. Schneider A, Hoyer H, Lotz B et al (2000) Screening for high-grade cervical intra-epithelial neoplasia and cancer by testing for high-risk HPV, routine cytology or colposcopy. Int J Cancer 89:529-534

40. Petry KU, Menton S, Menton M et al (2003) Inclusion of HPV testing in routine cervical cancer screening for women above 29 years in Germany: results for 8466 patients. Br J Cancer 88:1570-1577

41. Grouven U, Bender R, Ziegler A, Lange S (2007) Der Kappa-Koeffizient. Dtsch Med Wochenschr 132:e65-e68

42. Gerhardus A, Razum O (2010) A long story made too short: surrogate variables and the communication of HPV vaccine trial results. J Epidemiol Community Health 64:377-378

43. Kurzenhäuser S (2003) What kind of information do German health information pamphlets provide on mammography screening? Z Arztl Fortbild Qualitatssich 97:53-57

44. Gigerenzer G, Gray JAM (2011) Launching the century of the patient. In: Gigerenzer G, Muir JAM (Hrsg) Better doctors, better patients, better decisions: envisioning health care 2020. Strüngmann Forum Report, Bd 6. MIT, Cambridge, MA
45. Gigerenzer G, Mata J, Frank R (2009) Public knowledge of benefits of breast and prostate cancer screening in Europe. J Natl Cancer Inst 101:12161220

46. Schiffman M, Castle PE, Jeronimo J et al (2007) Human papillomavirus and cervical cancer. Lancet 370:890-907

47. Gerhardus A, Dören M, Gerlach FM et al (2009) Gebärmutterhalskrebs: Wie wirksam ist die HPV-Impfung? Dtsch Arztebl 106:330-334

48. Kristensen FB, Gerhardus A (2010) Health technology assessments: What do differing conclusions tell us? BMJ 341:c5236

49. Strobe-Statement (2007) Strengthening the reporting of observational studies in epidemiology. http://www.strobe-statement.org/

50. Elwyn GJ, O'Connor A, Stacey D et al (2006) Developing a quality criteria framework for patient decision aids: online international Delphi consensus process. BMJ 333(7565):417-424

51. Holmes-Rovner M (2007) International Patient Decision Aid Standards (IPDAS) beyond decision aids to usual design of patient education materials. Health Expect 10(2):103-107

52. O'Connor AM, Bennett CL, Stacey D et al (2009) Decision aids for people facing health treatment or screening decisions. Cochrane Database Syst $\operatorname{Rev}(3): C D 001431$

53. WHO (2010) Health information for patients and the general public produced by the German Institute for Quality and Efficiency in Health Care. A review by the World Health Organization 2008/2009. http://www.euro.who.int/_data/assets/pdf_file/0012/94989/E93735.pdf

54. Neumeyer-Gromen A, Lampert T, Stark K, Kallischnigg $G$ (2004) Disease management programs for depression - a systematic review and meta-analysis of randomized controlled trials. Med Care 42:1211-1221 